INRA Prod. Anim., 2004, 17 (5), 365-372
F. BATELLIER, M. GOVOROUN, J.P. BRILLARD

INRA, Station de Recherches Avicoles, F-37380 Nouzilly

Courriel :batellie@tours.inra.fr

\section{Sex-ratio chez les oiseaux sauvages et domestiques}

\begin{abstract}
Alors que le déterminisme du sexe chez les oiseaux comme chez tous les vertébrés est imposé par la répartition des chromosomes sexuels au cours de la méiose, on rencontre dans la nature, des exemples de sexratio déséquilibré. Quelles peuvent être les causes d'un tel déséquilibre ? Comment la femelle oiseau pourrait-elle contrôler le sex-ratio de sa descendance?
\end{abstract}

\begin{abstract}
Les oiseaux comme tous les vertébrés supérieurs sont des animaux à déterminisme sexuel chromosomique. Chaque parent transmet un chromosome particulier appelé « chromosome sexuel ». Ces chromosomes véhiculent les gènes majeurs de déterminisme du sexe qui conduiront au développement des testicules chez les mâles et des ovaires chez la femelle (en réalité seul l'ovaire gauche est fonctionnel chez les oiseaux). Dans les espèces aviaires, la femelle est hétérogamétique (ZW) et le mâle homogamétique (ZZ), une situation inversée par rapport à celle existant chez les mammifères (mâle hétérogamétique $\mathrm{XY}$ et femelle homogamétique XX). Dans les deux cas cependant, la stratégie de reproduction utilisée doit, selon la théorie mendélienne de la séparation aléatoire des chromosomes (autosomes et chromosomes sexuels) lors du passage des cellules diploïdes aux cellules haploïdes au cours de la méiose, conduire à l'obtention d'un nombre égal de gamètes porteurs de chacun des 2 chromosomes sexuels. Après la féconda-
\end{abstract}

\section{Résumé}

Les chromosomes sexuels déterminent le sexe des embryons chez les oiseaux comme chez tous les vertébrés supérieurs. Le sex-ratio de la descendance d'une population est donc, d'après la théorie mendélienne, équilibré, méiose oblige. On rencontre pourtant des contre-exemples à la fois dans les espèces d'oiseaux sauvages et domestiques. La femelle oiseau pourrait ainsi soit contrôler la répartition des chromosomes sexuels dans ses ovocytes, soit favoriser le développement des embryons d'un sexe donné. L'objet de cette revue est de préciser les conditions d'apparition de ce contrôle. Enfín, si ce déséquilibre est confirmé, la connaissance des mécanismes qui le régulent pourrait permettre à terme un contrôle du sexe des populations d'oiseaux. En production avicole, un tel contrôle fait l'objet d'un vieux rêve : orienter les lignées de poules pondeuses vers la production de femelles alors que les lignées de poulets de chairs ou les canards mulards seraient orientées vers la production de mâles. tion, on doit donc retrouver un nombre égal d'embryons mâles et femelles : le sex-ratio primaire, défini comme la proportion de mâles/femelles au moment de la fécondation est théoriquement égal à 1. Il existe pourtant, chez quelques espèces sauvages, des situations à partir desquelles un déséquilibre du sex-ratio a pu être observé. Les conditions particulières d'environnement ou bien des signaux spécifiques émis par le mâle sont mis en cause. De telles observations tendent à démontrer que dans certains cas, le déterminisme chromosomique du sexe ne serait pas seul impliqué dans le contrôle du sex-ratio. Des mécanismes faisant intervenir un « choix maternel » existent probablement. En dépit des critiques régulièrement émises à l'encontre de ce type d'observations au cours des dernières décennies (Williams 1979), les travaux contribuant à les valider se multiplient alors que le mécanisme du déterminisme du contrôle par la femelle se précise. Dans une intéressante revue, Sheldon (1998) commente les différences de répartition entre sexes dans une population donnée puis la répartition du sex-ratio dans cette même population. Il indique qu'une sélection impliquant des facteurs environnementaux agirait sur la répartition des sexes dans la population. Sheldon précise enfin que si les oiseaux sont comme les autres espèces, contraints, méiose oblige, de produire un nombre identique de mâles et de femelles lors de la fécondation, la seule adaptation restant possible est « l'élimination sélective » de l'un des deux sexes. Dans ce cas, on peut seulement observer un déséquilibre du sex-ratio secondaire (proportion de mâles/femelles au moment de l'éclosion) ou tertiaire (proportion de mâles/femelles à l'âge de la maturité sexuelle). Cette «élimination » diminue toutefois la valeur reproductive des 
portées, et augmente le « coût » de cette stratégie, ce qui explique son caractère exceptionnel ! Sheldon résume ainsi le problème qui se pose à la femelle oiseau : elle doit produire un sex-ratio optimal en réponse à la disponibilité en nourriture des semaines ou mois futurs ou bien en réponse à une stimulation induite par des mâles présentant un phénotype particulier (parure sexuelle). On comprend bien que la probabilité d'une estimation correcte du rapport coût/efficacité soit très faible dans ces conditions.

Dans les espèces d'oiseaux domestiques, malgré un grand nombre d'études, la conclusion la plus communément admise (basée sur des études de croisements génétiques) est qu'il n'existe pas de différence de répartition du sex-ratio. Le contrôle du sexe dans ces espèces à fort intérêt économique est pourtant à l'origine d'un vieux rêve qui consisterait, par exemple, à ne produire que des femelles dans les souches de poules pondeuses alors que les souches destinées à la production de viande ne produiraient que des mâles....

L'objectif de cette revue est de préciser les conditions dans lesquelles les femelles oiseaux sont susceptibles de modifier l'équilibre du sex-ratio dans leur descendance, en distinguant bien les modifications du sexratio primaire de celles du sex-ratio secondaire. Nous avons choisi de séparer les études portant sur les oiseaux sauvages et les oiseaux domestiques. Les différences qui existent à la fois dans les modes de vie de ces animaux et dans les approches expérimentales utilisées par les équipes de recherche sont en effet très importantes. Les oiseaux sauvages se prêtent par exemple mal à l'étude du sex-ratio primaire, il est en effet difficile d'avoir accès aux œufs sitôt après la fécondation, ou aux ovocytes avant la fécondation.

Depuis quelques années, les études du sexratio primaire se sont développées grâce aux techniques de sexage moléculaire ne nécessitant que des quantités très faibles de matériel biologique embryonnaire. Nous avons nousmêmes utilisé ce type d'approche pour étudier les variations de sex-ratio à plusieurs stades de l'incubation chez des canards Pékin, Barbarie ou mulards (Batellier et al 2004). Les risques d'erreurs restent néanmoins présents notamment lors de l'utilisation de la PCR pour le sexage d'un embryon à un stade très précoce, ou pour la détection du chromosome sexuel porté par l'ovocyte avant la fécondation (Arnold et al 2003). D'après cette étude, les très faibles quantités d'ADN et les possibilités de contamination par du matériel parental rendent aléatoire toute estimation du sex-ratio primaire réalisée sur cette seule base.

\section{1 / Répartition du sex-ratio des descendants chez les oiseaux sauvages}

En règle générale, le sex-ratio des descendants d'oiseaux sauvages est équilibré mais un sex-ratio tertiaire déséquilibré favorisant soit les mâles soit les femelles a été depuis longtemps décrit chez plusieurs espèces d'oiseaux sauvages (Mayr 1939). Des observations détaillées le conduisent à penser que ces déséquilibres sont présents dès le sexratio primaire, puisque la mortalité ne semble jamais liée au sexe quelque soit l'âge des oiseaux. Il semble que ce déséquilibre soit une réponse des oiseaux à une modification de leurs conditions de vie. Dès cette époque, des facteurs « secondaires » non chromosomiques sont suspectés intervenir sur l'ovocyte avant l'ovulation, pour orienter le sexe du futur embryon avant la fécondation. Nous avons résumé ci-après les principales causes avancées pour expliquer ce type de déviation observé depuis dans plusieurs espèces.

\section{1 / Influence de l'environnement externe}

En manipulant les conditions de vie de la mouette à dos noir (Larus fuscus), Nager et al (1999) ont montré que l'état physique des femelles influence le sex-ratio de ses descendants, par exemple en augmentant la mortalité des mâles issus de femelles sous-alimentées. Progressivement, les femelles orientent alors le sex-ratio (secondaire) de leur descendance (évalué par prélèvement sanguin puis sexage par PCR à l'éclosion) vers la production d'autres femelles dont la survie n'est pas affectée par la restriction alimentaire. Ces résultats montrent que dans cette espèce, les femelles ont la capacité d'ajuster le sex-ratio de leur descendance aux conditions d'environnement maternel présentes au moment de la fécondation. Un autre exemple de l'incidence de l'environnement externe sur le sex-ratio des descendants a été démontré chez la fauvette aquatique (Acrocephalus paludicola), un petit passereau insectivore chez lequel les mâles sont de taille plus importante que les femelles. Dans cette espèce dont l'habitat inclut aujourd'hui le Bélarus, la Pologne et l'Ukraine, Dyrcz et al (2004) ont pu mettre en évidence une plus grande proportion de femelles dans les couvées de grande taille soumises à des températures basses juste avant la période de ponte (par sexage moléculaire basé sur la détection du gène CHD après prélèvement sanguin réalisé entre les jours 8 et 11 après l'éclosion de la première couvée de la saison de chaque femelle. Le gène CHD est spécifique des chromosomes sexuels, après PCR et électrophorèse, les mâles présentent une bande et les femelles 2 bandes) une plus grande proportion de femelles dans les couvées de grande taille soumises à des températures basses juste avant la période de ponte. Pour ces auteurs, l'explication d'une telle déviation du sex-ratio secondaire, tient au fait que des conditions météorologiques défavorables pénalisent surtout les individus qui, dans une portée, sont aussi les plus exigeants et les plus « coûteux » en nourriture, c'est-à-dire ceux de plus grande taille. Ce type d'observation a d'ailleurs été rapporté dans plusieurs autres espèces exprimant un dimorphisme sexuel marqué. Un autre travail conduit chez la chouette hulotte par Appleby et al (1997) démontre l'incidence 
de la densité de population sur le sex-ratio, une proportion plus importante de poussins femelles étant recensée dans les nichées issues de territoires où l'espèce est présente en fortes densités. Les fragments microsatellites spécifiques de la femelle ont été analysés à partir de prélèvements sanguins réalisés entre 1 et 21 jours après l'éclosion. Les auteurs considèrent avoir étudié le sex-ratio primaire car seules les couvées pour lesquelles tous les poussins ont éclos ont été analysées, l'éventuel biais induit par une mortalité embryonnaire liée au sexe est ainsi évité.

Dans une étude portant sur cinq espèces de rapaces diurnes, Daan et al (1996) considèrent que si le sex-ratio de la descendance est rarement dévié, on observe souvent un lien entre le moment de l'année et la production préférentielle de l'un ou l'autre des sexes. Ils ont ainsi montré un lien entre couvée tardive et sex-ratio biaisé en faveur des mâles dans trois des espèces étudiées et entre couvée tardive et sex-ratio biaisé en faveur des femelles pour les deux autres espèces. Or, dans les trois premières espèces, les femelles se reproduisent intensivement pendant leur première année de vie puis la taille des couvées diminue ensuite alors que la fertilité des mâles, dans ces mêmes espèces, ne semble pas affectée par l'âge. Dans les deux autres espèces, le système est inversé : les mâles se reproduisent pendant leur première année de vie puis beaucoup moins, alors que les femelles ne semblent pas affectées par l'âge. Ces auteurs concluent en une adaptation du sex-ratio à la saison pour chaque espèce en fonction de l'âge de la puberté pour chacun des sexes. Le sexe qui a un retard à la puberté sur l'autre sera produit de manière plus importante plus tôt dans la saison. Le modèle de simulation décrit prend également en compte la baisse de fertilité de femelles en fin de saison de reproduction.

\section{2 / Influence de l'environnement social}

La fauvette des Seychelles (Acroephalus sechellensis) est un petit oiseau endémique dans quelques îles de cet archipel. Dans cette espèce, les filles aident les parents à élever la génération suivante alors que les fils se dispersent. Lorsque l'environnement est favorable (riche en insectes) une plus grande proportion de femelles ( $87 \%$ ) voit le jour et les parents sont bien aidés pour abondamment nourrir leur descendance. A l'inverse, lorsque l'environnement devient hostile, le sex-ratio secondaire se déséquilibre en faveur des mâles (77\%) qui tirent un meilleur parti de la compétition pour la nourriture (Komdeur et al 1997). Les auteurs assurent que la différence est bien due à un déséquilibre du sex-ratio primaire mais seuls des oisillons de 4 à 12 jours ont été sexés sans indication sur une éventuelle mortalité embryonnaire. Les observations de Kilner (1998) chez le Zebra finch confortent le travail précédent en indiquant, elles aussi, une déviation possible du sex-ratio secondaire et tertiaire de l'espèce conditionné par la dispo- nibilité en nourriture (évalué dès l'éclosion par l'observation d'une particularité du plumage liée au sexe, puis confirmé à l'âge adulte). Quand la nourriture manque, le sex-ratio secondaire devient favorable aux mâles mais la mortalité embryonnaire des femelles augmente (et vice-versa quand la nourriture est abondante). Le sex-ratio primaire ne semble donc pas affecté dans cette espèce. Il semble que le sexe des animaux les plus gros au moment où les jeunes quittent le nid soit celui dont la production est diminuée quand la nourriture manque (par une augmentation de sa mortalité embryonnaire).

Dans plusieurs autres espèces, il apparaît que les descendants du sexe qui aide les parents sont produits en plus grande quantité que ceux du sexe opposé. Ainsi en est-il du pic à cocarde rouge (Picoides borealis) dont le sex-ratio des nichées (observation des tâches différentes entre mâles et femelles visibles à partir de 15 jours après l'éclosion) a été étudié par Gowaty et al (1985). Ces auteurs concluent, tout comme Emlen et al (1986), que le sexe apportant une aide parentale « rembourse » en quelque sorte une partie de l'effort de production fourni par les parents pour se reproduire.

Un autre exemple de déviation du sex-ratio chez des espèces sauvages est celui du perroquet Electus (Electus rorschach), capable de produire des séries exclusives de mâles pouvant atteindre 30 individus sans que l'on puisse expliquer les mécanismes en cause (Heinsohn et al 1997). Les auteurs considèrent que la déviation du sex-ratio existe dès la fécondation, malgré un sexage réalisé au moment où les oisillons sont emplumés. Dans cet exemple précis, lorsqu'un seul oisillon éclot, on observe sur l'ensemble des couvées un sex-ratio équilibré (au moment de l'emplumement), par contre le sex-ratio est déséquilibré si on observe la descendance de chaque femelle. Ce qui implique, un rôle actif de la femelle pour le contrôle du sexe de ses descendants. Les 2 hypothèses retenues sont : soit un contrôle au moment de la fécondation (évalué par modèle mathématique statistique), soit une élimination sélective d'un des 2 sexes par la femelle après l'éclosion. La seule information dont on dispose sur le mode de vie de cet oiseau (et qui le rapproche de la fauvette précédemment citée) est que mâles et femelles s'associent pour élever les jeunes (comportement unique chez les perroquets).

\section{3 / Influence des caractéristiques physiques parentales sur le sex-ratio des descendants}

L'environnement fourni par les parents à leur descendance influence leur capacité à se reproduire et peut donc affecter le sex-ratio (Trivers et Willard 1973). En dehors du rôle joué par l'état physique maternel (voir plus haut), d'autres particularités physiques tels que l'importance des caractères sexuels secondaires du mâle peuvent influencer les performances de reproduction. Ainsi, un mâle ayant des caractères sexuels bien visibles 
aura par exemple de meilleures performances de reproduction qu'un mâle à caractères sexuels secondaires peu marqués (Ellegren et al 1996). Des manipulations expérimentales sur de petits oiseaux sauvages (Ficedula albicolis) ont permis de mettre en évidence que plus la tache sur la tête des mâles est grande, plus le nombre de mâles dans leur descendance sera élevé (Ellegren et al 1996). Cette tache sur la tête représente un " atout » et permet aux mâles un plus grand choix des femelles. Il est possible que la taille de cette tache soit liée à d'autres caractéristiques sexuelles. Aucune explication des mécanismes de contrôle du sex-ratio par la femelle n'est fournie dans cet article.

\section{2 / Distribution du sex-ratio chez les oiseaux domestiques}

A l'encontre des espèces sauvages, il n'existe que peu d'études faisant état d'un déséquilibre du sex-ratio dans les espèces aviaires domestiques. Une synthèse ancienne de Byerly et Jull (1935) traitant de cette question rapporte que dans les cas où un sex-ratio est déséquilibré, l'on observe toujours une plus forte mortalité des femelles, ce qui indique que seul le sex-ratio secondaire (distribution des sexes au cours de la vie embryonnaire) serait concerné. Les auteurs précisent toutefois qu'une incertitude persiste quant au sexe des embryons morts avant que leur sexe puisse être déterminé par un examen macroscopique. Dans un cas, Byerly et Jull (1935) rapportent un déficit génétique lié au sexe (chondrodystrophie) ayant entraîné une mortalité plus importante de poulets mâles dans une souche (Rhode Island Red), aucune différence n'étant observée dans l'autre souche testée (White Leghorn).

Par ailleurs, plusieurs études décrivent l'existence de souches particulières de poules ayant un sex-ratio primaire déséquilibré. Ichinoe (1973) s'est intéressé à la relation entre le moment d'éclosion (séquencé par tranches de 3 heures) et le sex-ratio dans 6 croisements de poulets. Il apparaît que les femelles éclosent en moyenne plus tôt que les mâles. La différence est très significative dans 4 des 6 croisements étudiés mais n'apparaît pas dans les 2 autres. Lâge des reproducteurs n'affecte pas cette répartition.

Hays a publié la première étude du sex-ratio primaire des oiseaux domestiques en 1945. Cet auteur cite également plusieurs travaux ayant porté sur le sex-ratio secondaire (Landauer et Landauer 1931), aucun d'entre eux ne faisant apparaître une quelconque déviation chez le poulet. Afin d'explorer avec précision les distributions de sex-ratio primaire et en l'absence d'outils performants tels que ceux dont nous disposons aujourd'hui, Hays a utilisé des poules $(n=39)$ qui produisent $100 \%$ d'œufs fertiles et sans aucune mortalité embryonnaire pendant au moins 2 mois de suite. Un total de 931 descendants fut obtenu parmi lesquels 61 moururent avant sexage. Des 870 survivants, 432 étaient des mâles et 438 des femelles. Ces résultats indiquent clairement une répartition équilibrée du sex-ratio à l'éclosion (sex-ratio secondaire) qui laisse penser que le sex-ratio primaire (à la fécondation) était lui-même équilibré. Lors de travaux précédents conduits eux aussi chez le poulet, le même auteur avait décrit une mortalité embryonnaire (Hays 1949, Hays et Spear 1950) et une mortalité périnatale (Hays 1951) plus importantes chez les femelles que chez les mâles. Sur la base d'observations complémentaires, il apparaît aussi que les troupeaux âgés ont également une tendance à induire une déviation du sexratio en faveur des mâles à cause d'une augmentation de la mortalité embryonnaire des embryons femelles (Hays et Spear 1952, Hays 1952). Parmi les facteurs susceptibles d'influencer le sex-ratio chez le poulet, Hays souligna particulièrement le rôle du taux d'éclosion et celui de l'âge des mâles. Comme si après un certain âge, le pouvoir fécondant des mâles devenait plus faible pour les « œufs devant donner des femelles »... Ce même auteur démontra enfin que les mâles des familles à sex-ratio fortement déviée transmettaient souvent une anomalie létale liée au sexe à leur descendance.

Une autre approche développée par Landauer (1957) chez le poulet a eu pour but de préciser la répartition du sex-ratio primaire ainsi que sa variabilité entre femelles ayant des origines génétiques diverses. Des deux souches analysées (Rhodes Island et Black Minorca), aucune n'a exprimé de déviation du sex-ratio. L'auteur conclut à l'équilibre de ce caractère dans l'espèce ainsi qu'à l'absence de contrôle exercé par les gènes autosomaux sur le déterminisme du sexe chez les oiseaux.

Plus récemment, Klein et al (2002) se sont intéressés à la distribution la plus précoce du sex-ratio au cours d'une série de ponte chez la poule. Rappelons ici que, le sexe de l'embryon d'oiseau est fixé au cours de la maturation folliculaire et plus précisément au cours de la première division méiotique de l'ovocyte. Chez la poule, cette première division se déroule environ une heure avant l'ovulation (à ce stade qui précède l'expulsion du premier globule polaire, la réduction haploïde du génome et le partage des chromosomes sexuels sont donc achevés). Dans leur étude Klein et al (2002) ont adapté une technique de diagnostic du sexe très précoce, utilisable même sur un ovocyte car permettant de travailler à partir d'une seule cellule (amplification d'une séquence répétitive du gène Xho1, spécifique du chromosome W). Ils ont utilisé cette technique pour évaluer le sexe primaire de couvées individuelles dans deux souches de poules en début de saison de reproduction (Leghorn, une souche ponte de grande diffusion et Deutsche Zwerghühner, une souche ancienne de collection). La distribution du sex-ratio des 5 premiers oufs de série a été confrontée au « sex-ratio » des oufs infertiles (ou sexe déterminé par l'ovocyte d'un ouf infertile) ainsi qu'à celle des embryons morts précocement. Si aucun effet souche ne fut mis en évidence, il est apparu que les 5 premiers oufs pondus contenaient, fécondés ou non, une proportion plus élevée de mâles mais que, parmi les œufs fécondés, le taux de mortalité était plus élevé chez les embryons 
mâles que chez les femelles lors des 3 premiers jours d'incubation. Cette déviation du sex-ratio primaire, fortement variable d'une poule à l'autre a eu tendance à décroître au cours de l'avancement en ponte. Aucune déviation n'a pu être formellement mise en évidence, bien que pour les auteurs de ce travail, de nouvelles études sont nécessaires avant de conclure.

Un cas particulier de déséquilibre du sexratio secondaire est illustré par nos propres observations conduites chez des canetons mulards à l'éclosion (Batellier et al 2004). Le croisement « mulard » est issu du croisement interspécifique entre un canard mâle Barbarie et une femelle Pékin. Or, dans les couvoirs industriels produisant les canetons mulards, les proportions de canetons de chaque sexe, variables d'une incubation à l'autre mais corrélées à la fertilité initiale des œufs (résultats non publiés), sont favorables aux mâles (60$65 \%$ du total des canetons éclos). Dans notre approche, nous avons cherché à savoir si ce déséquilibre était dû à une déviation du sexratio primaire ou bien s'il résultait d'une mortalité plus forte des embryons femelles. Quatre origines ou croisements ont été étudiés : Pékin, Barbarie, mulards et mulards inversé (« hinny »). Les embryons ayant

Tableau 1. Comparaison des méthodes de détermination du sex-ratio dans les différentes études.

\begin{tabular}{|c|c|c|c|c|}
\hline Auteurs & Type de sex-ratio & Âge au sexage & Technique utilisée & Biais supprimé ? \\
\hline $\begin{array}{l}\text { Gowaty } \\
\text { et al } 1985\end{array}$ & $\begin{array}{l}\text { Secondaire } \\
\text { et tertiaire }\end{array}$ & $\begin{array}{c}15 \text { jours et + } \\
\text { après l'éclosion }\end{array}$ & $\begin{array}{l}\text { Tâches de plumage } \\
\text { différentes selon le sexe }\end{array}$ & \\
\hline $\begin{array}{l}\text { Kilner } \\
1998\end{array}$ & $\begin{array}{l}\text { Secondaire } \\
\text { et tertiaire }\end{array}$ & $\begin{array}{l}\text { À l'éclosion } \\
\text { et à } 30 \text { jours } \\
\text { (séparation } \\
\text { des parents) }\end{array}$ & $\begin{array}{l}\text { Plumage différent } \\
\text { selon le sexe }\end{array}$ & $\begin{array}{c}\text { Oui, mais parle } \\
\text { de sex-ratio primaire } \\
\text { et secondaire au lieu } \\
\text { de secondaire et tertiaire }\end{array}$ \\
\hline Nager et al 1999 & Secondaire & éclosion & Sexage par PCR & \\
\hline $\begin{array}{c}\text { Dyrcz } \\
\text { et al } 2004\end{array}$ & Secondaire & $\begin{array}{c}8 \text { à } 11 \text { jours } \\
\text { après l'éclosion }\end{array}$ & $\begin{array}{l}\text { Sexage par PCR } \\
\text { (détections de CHD) }\end{array}$ & \\
\hline $\begin{array}{l}\text { Batellier } \\
\text { et al } 2004\end{array}$ & $\begin{array}{c}\text { Primaire } \\
\text { et secondaire }\end{array}$ & $\begin{array}{c}1 \text { et } 5 \text { jours d'incubation } \\
\text { d'incubation } \\
\text { puis à l'éclosion }\end{array}$ & Sexage par PCR & \\
\hline $\begin{array}{l}\text { Appleby } \\
\text { et al } 1997\end{array}$ & Secondaire & $\begin{array}{l}1 \text { à } 21 \text { jours } \\
\text { après l'éclosion }\end{array}$ & $\begin{array}{c}\text { Analyse des fragments } \\
\text { microsatellites } \\
\text { spécifiques de } \mathrm{W} \\
\end{array}$ & $\begin{array}{l}\text { Évité car le sexe des } \\
\text { poussins morts avant } \\
\text { l'éclosion est étudié }\end{array}$ \\
\hline $\begin{array}{l}\text { Komdeur } \\
\text { et al } 1997\end{array}$ & Secondaire & 4 à 12 jours & $\begin{array}{c}\text { Amplification au } \\
\text { hasard de marqueurs } \\
\text { d'ADN polymorphe (RAPD) }\end{array}$ & $\begin{array}{c}\text { Oui selon les auteurs } \\
\text { mais pas d'informations } \\
\begin{array}{c}\text { sur la mortalité } \\
\text { embryonnaire }\end{array}\end{array}$ \\
\hline $\begin{array}{c}\text { Klein } \\
\text { et al } 2002\end{array}$ & Primaire & $\begin{array}{l}\text { Embryon d'une } \\
\text { seule cellule et } \\
\text { avant fécondation }\end{array}$ & $\begin{array}{l}\text { Amplification de Xho1 } \\
\text { spécifique } \\
\text { du chromosome W }\end{array}$ & $\begin{array}{c}\text { Biais possible } \\
\text { par analyse } \\
\text { de contaminants maternels }\end{array}$ \\
\hline
\end{tabular}

Tableau 2. Exemples de travaux montrant des cas de sex-ratio déséquilibrée (espèces d'oiseaux sauvages et domestiques) et causes de ce déséquilibre.

\begin{tabular}{|c|c|c|c|}
\hline & Espèce étudiée & $\begin{array}{l}\text { Déséquilibre en faveur } \\
\text { des mâles ou femelles }\end{array}$ & Cause du déséquilibre \\
\hline $\begin{array}{c}\text { Nager } \\
\text { et al } 1999\end{array}$ & Mouette à dos noir & Femelles & Sous-alimentation des mères \\
\hline $\begin{array}{c}\text { Dyrcz } \\
\text { et al } 2004\end{array}$ & Fauvette aquatique & Femelles & $\begin{array}{l}\text { Basses températures } \\
\text { juste avant la ponte }\end{array}$ \\
\hline $\begin{array}{l}\text { Appleby } \\
\text { et al } 1997\end{array}$ & Chouette hulotte & Femelles & Forte densité de population \\
\hline $\begin{array}{c}\text { Daan } \\
\text { et al } 1996 \\
\end{array}$ & $\begin{array}{c}5 \text { espèces } \\
\text { de rapaces diurnes }\end{array}$ & Varie selon l'espèce & Âge à la puberté \\
\hline $\begin{array}{l}\text { Komdeur } \\
\text { et al } 1997\end{array}$ & $\begin{array}{c}\text { Fauvette } \\
\text { des Seychelles }\end{array}$ & Varie & Quantité de nourriture \\
\hline $\begin{array}{c}\text { Kilner } \\
1998\end{array}$ & Zebra finch & Varie & Quantité de nourriture \\
\hline Ichinoe 1973 & Poule & Femelles & Heure d'éclosion \\
\hline Hays 1952 & Poule & Mâles & Âge avancé des reproducteurs \\
\hline $\begin{array}{l}\text { Batellier } \\
\text { et al } 2004\end{array}$ & $\begin{array}{c}\text { Canard mulard } \\
\text { et autres croisements }\end{array}$ & Mâles & $\begin{array}{l}\text { Croisement interspécifique } \\
\text { surmortalité des femelles }\end{array}$ \\
\hline
\end{tabular}


1 et 5 jours d'incubation ont été sexés par PCR alors que les embryons plus âgés et les canetons ont été sexés par observation macroscopique. Aucune différence significative du sex-ratio primaire n'a été mise en évidence (jours 1 ou 5) quelque soit le croisement étudié (figure 1). Par contre, nos résultats ont confirmé la déviation du sexratio à l'éclosion (sex-ratio secondaire) chez les mulards et établi l'existence d'une déviation comparable chez les mulards inverses. En complément, nous avons mis en évidence chez les mulards que la population de canetons dits « non commercialisables » $\mathrm{au}$ moment de l'éclosion n'était composée que de $32 \%$ de mâles, une proportion plus forte de femelles sont donc éliminées à ce moment. De plus, chez les canards mulards, on observe une corrélation entre le taux d'éclosion et la fertilité des troupeaux. Ce plus fort taux d'éclosion s'accompagne d'un sex-ratio plus équilibré. Les femelles conçues sont donc capables d'éclore quand la fertilité du troupeau est bonne. Ceci laisse à penser que dans ce croisement la qualité initiale des gamètes mâles ou femelles exerce non seulement un rôle direct sur la fertilité mais qu'elle conditionne aussi la qualité des embryons et leur aptitude à se développer normalement jusqu’à l'éclosion.

Nous allons enfin évoquer les techniques d'étude des caryotypes et leurs évolutions possibles. Fechheimer (1970) et Dinkel et al (1978) ont pu démontrer que le sex-ratio primaire ( $16^{\text {ème }}$ heure d'incubation) est équilibré dans plusieurs lignées de poules pondeuses ou de chair. Ces auteurs réalisent un caryotype très précoce, qui permet de déterminer la présence d'un ou de deux chromosomes Z. Depuis une vingtaine d'années environ, la possibilité d'accéder à des techniques de Cbanding de l'hétéro chromatine a permis de « visualiser» le chromosome W. Ce type d'approche a permis de confirmer l'absence de déviation du sex-ratio dans plusieurs lignées de poules (De Boer et al 1984).

\section{Conclusion}

Parmi les différences d'approches utilisées (tableau 1) pour étudier les déviations éventuelles de sex-ratio chez des oiseaux, il apparaît que les travaux réalisés sur espèces sauvages sont en premier lieu basés sur l'étude individuelle de chaque couvée. Une telle approche est sans doute adaptée lorsqu'il s'agit d'expliquer les mécanismes permettant à chaque femelle de contrôler le sexe de ses descendants. On peut remarquer que les résultats sont souvent contradictoires à cause des faibles effectifs étudiés. A l'inverse, dans les espèces domestiques ayant un fort intérêt économique, on étudie pour un croisement donné, le déséquilibre du sex-ratio de l'ensemble d'une production sans aborder l'approche individuelle. De plus, il est difficile dans ces études de distinguer ce qui peut avec certitude être compris comme une estimation du sex-ratio primaire de ce qui fait en réalité référence à une estimation du sex-ratio secondaire (tableau 2).

En dépit des limites d'utilisation inhérentes à des prélèvements très précoces du matériel biologique (Arnold et al 2003), les techniques de biologie moléculaire dont on dispose aujourd'hui permettent, dans des conditions d'utilisation optimales, de différencier le sexratio avec une précision suffisante pour éliminer la mortalité embryonnaire précoce comme cause d'un déséquilibre (Appelby et al 1997, Komdeur et al 1997). Sur la base de plusieurs des observations rapportées ci-dessus, les femelles de quelques espèces seraient donc capables (par un mécanisme restant inconnu) de contrôler la sélection des chromosomes au moment de la méiose ! Pour expliquer le fonctionnement d'un tel mécanisme, une résorption sélective des ovocytes du sexe non désiré a même été envisagée (Emlen et al 1986). Il reste que dans les différentes situations précitées, des preuves directes établissant sans discussion possible le contrôle par la mère du sex-ratio primaire de ses cou-

Figure 1. Exemple de gel d'électrophorèse d'ADN génomique amplifié par PCR "multiplex" (une amorce spécifique de chromosome $W$ et une amorce spécifique du gène de la fibrilline).

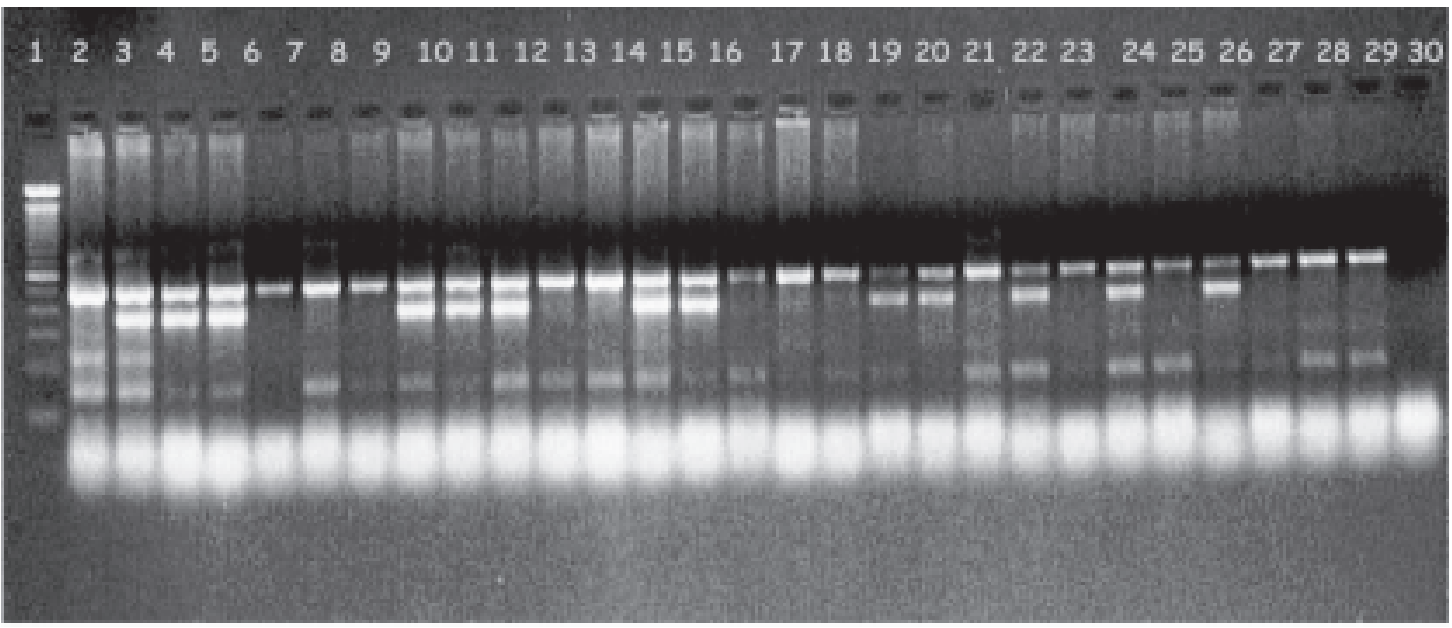

Dans le puits $n^{\circ} 1$, le marqueur de poids moléculaire a été déposé.

Dans le puits $n^{*} 30$, seule de l eau a ete déposte (temoin negatif).

Dans les puits $n^{*} 2$ a $n^{\circ} 29$, les échantillons de matériel embryonnaire ont été déposés. La première bande en haut est celle du gène de la fibrilline, la seconde bande est celle spécifique du chromosome W. Les embryons ayant 1 seule bande sont donc des malles et ceux ayant 2 bandes, des fernelles. 
vées restent à établir. Or, un tel travail n'est pas aisé car, aux outils dont nous disposons actuellement en biologie moléculaire (PCR, FISH...), il faut associer un protocole de prélèvements extrêmement précis ayant de plus une puissance statistique suffisante, deux conditions que les contraintes imposées par des modèles expérimentaux sauvages rendent particulièrement délicates à respecter...

Sheldon (1998) pose plusieurs questions essentielles sur les éventuels mécanismes de contrôle du sex-ratio primaire : comment une femelle oiseau peut elle contrôler la ségrégation des chromosomes sexuels lors de la méiose ? Peut-il se produire une résorption sélective des ovocytes du « mauvais » sexe ? La femelle attend t-elle un ovocyte du «bon » sexe pour commencer à couver? Heinsohn et al (1997) ont montré que le déséquilibre observé chez le perroquet est présent dès l'oviposition et leurs observations renforcent l'hypothèse selon laquelle les femelles de cette espèce peuvent contrôler le sex-ratio de leurs descendants. Le même contrôle par « voie méiotique » est suggéré par Kilner (1998) qui a fait varier expérimentalement la quantité de nourriture disponible et vérifié dans ses expériences, les théories de Trivers et Willard (1973) selon lesquelles les femelles en bonne condition physique produisent plus de mâles et les femelles en mauvaise condition physique diminuent le taux de mâles dans leur descendance. Chez le canari et le Zebra finch, le contrôle du sex-ratio serait effectué par la femelle dont les variations du taux de testostérone plasmatique influenceraient le follicule préovulatoire puis ainsi le sexe des embryons (Schawbl, 1993). Müller et al (2002) propose de retenir cette hypothèse mais minimise son rôle dans le déterminisme du sexe de l'embryon. Il montre par contre que les variations de taux des hormones circulantes (ex : testostérone) dépendent du statut social des femelles, les « dominantes » (les œufs des poules dominantes et contenant des embryons mâles présentent une concentration plus forte en testostérone) ayant une descendance majoritairement «mâle » alors que les femelles « dominées » (les œufs des poules dominées et contenant des embryons femelles ont une concentration plus forte en testostérone) ont plus de descendants femelles. Cet auteur conclut à l'existence d'un mécanisme adaptatif grâce auquel les femelles favoriseraient, en augmentant la concentration en testostérone dans les œufs contenant des embryons de l'un ou l'autre sexe, la production de l'un des 2 sexes selon leur propre statut social. Le mécanisme mis en cause reste cependant à déterminer.

Van Middelkoop et Lourens (2002) ont récemment étudié les effets de la température d'incubation sur l'éclosabilité des œufs de poule selon le sexe des embryons. Ils ont montré une plus grande sensibilité et donc une mortalité embryonnaire supérieure liée à une augmentation modérée de la température d'incubation chez les embryons femelles.

Une des hypothèses avancées pour expliquer cette fragilité des embryons femelles est d'origine cytogénétique. Le chromosome Z n'est présent qu'en une seule copie chez la femelle qui possède par contre un chromosome $\mathrm{W}$ de structure très particulière. Le chromosome W est en effet majoritairement constitué d'hétéro chromatine (Stefos et Arrighi, 1971) et contient entre 70 et $90 \%$ de séquence répétitives, notamment $X h o I$ gène spécifique du chromosome W, identifié par Saitoh et al (1991). Le chromosome W contient des gènes qui ne sont pas encore tous identifiés et dont les rôles ne sont pas définis. On ne sait donc pas encore actuellement, si la présence d'une seule copie des gènes présents sur le chromosome $\mathrm{Z}$, fragilise les femelles et si le relais de l'expression de ces gènes peut être assuré par le chromosome W. Notamment, ATP5A1 et CHD1 sont attribués au chromosome $\mathrm{W}$ mais seraient également présent sous forme d'une copie très proche sur le chromosome Z (Fridolfsson et al 1998).

Il reste qu'aucune extrapolation à partir de connaissances établies chez les mammifères n'est possible à ce niveau à cause du manque d'homologie entre les chromosomes $\mathrm{W}$ de la femelle d'oiseau et Y du mâle de mammifère. Ainsi, les quelques gènes identifiés (Fridolfsson et al 1998) sur le chromosome W des oiseaux ne sont pas liés au sexe chez les mammifères.

En conclusion, nous revenons à la remarque de West et al (2002) qui suggèrent que le principal problème posé au chercheur s'intéressant au sex-ratio est d'élucider comment et pourquoi certains organismes sont capables d'ajuster le sex-ratio de leur descendance avec autant de précision alors que le déterminisme du sexe est sous contrôle de chromosomes sexuels distribués d'une manière que l'on pensait jusqu'à ce jour aléatoire au cours de la méiose.

Cette recherche peut conduire à terme à la maîtrise du sex-ratio d'une production animale. Ce problème est particulièrement important chez les oiseaux dans plusieurs types de productions. En effet dans plusieurs productions la moitié des poussins est éliminée dès l'éclosion : les poussins mâles des souches «pondeuses» (on ne garde que les femelles), les poussins femelles des souches « chair ", les canetons femelles des souches «mulard» (seuls les mâles sont aptes à être gavés pour la production de foie gras). Dans tous ces cas, la maîtrise du sex-ratio des poussins, actuellement encore impossible, serait pourtant une source d'un gain économique significatif. 


\section{Références}

Appleby B.M., Petty S.J., Blakey J.K., Rainey P., Macdonald D.W., 1997. Does variation of sex ratio enhance reproductive success of offspring in tawny owls (Strix aluco)? Proc. R. Soc. B., 264, 1111-1116.

Arnold K.E., Orr, K.J., Griffiths R., 2003. Primary sex ratios in birds : problems with molecular sex identification of undeveloped eggs. Mol. Ecol., 12(12), 3451-3458.

Batellier F., Marchal F., Scheller M.F., Gautron J., Sellier N., Taouis M., Monbrun C., Vignal A., Brillard J.P., 2004. Sex ratios in mule duck embryos at various stages of incubation. Theriogenology, 61, 573-580.

Byerly T.C., Jull M.A., 1935. Sex ratio and embryonic mortality in the domestic fowl. Poult. Sci., 14, 217-220.

Daan S., Dijkstra C., Weissing F.J., 1996. An evolutionary explanation for seasonal trends in avian sex ratios. Behav. Ecol., 7, 426-430.

De Boer L.E.M., De Tag T.A.G., Frankenhuis M.T. Zonneveld A.J., Sawevelt J., Belterman R.H.R., 1984. Triploïdy in Gallus domesticus embryos, hatchlings and adult intersex chickens. Genetica, 65, 83-87.

Dinkel B.J., Fecheimer N.S., 1978. Cytogenetic analysis of highly inbred lines of chikens. Poult. Sci., 57, 548-549.

Dyrcz A., Sauer-Gürth, Tkadlec E., Wink M., 2004. Offspring sex ratio variation in relation to brood size and mortality in a promiscuous species : the Aquatic Warbler Acrocephalus paludicola. Ibis, 146, 269-280.

Ellegren H., Gustafsson L. , Sheldon B.C., 1996. Sex ratio adjustment in relation to paternal attractiveness in a wild bird population. Proc. Natl. Acad. Sci. USA, 93, 11723-11728.

Emlen S.T., Emlen J.M., Levin S.A., 1986. Sex ratio selection in species with helpers-at-the-nest. Am. Nat., 127, 1-8.

Fecheimer N.S., 1970. Sex proportion of domestic chicken at 16 hours of incubation. J. Reprod. Fert.; 23, 363-369.

Fridolfsson A.K., Cheng H., Copeland N.G., Jenkins N.A., Liu H.C., Raudsepp T., Woodage T., Chowdhary B. Halverson J., Ellegren H., 1998. Evolution of the avian sex chromosome from an ancestral pair of autosomes. Proc. Natl. Acad. Sci USA, 95, 8147-8152.

Gowaty P.A., Lennartz M.R., 1985. Sex ratios of nestlings and fledging red cockaded woodpeckers (Picoides borealis) favour males. Am. Nat., 126, 347-353.

Hays F.A., 1945. The primary sex ratio in domestic chickens. Am. Nat., 79, 184-186.

Hays F.A., 1949. Sex influence on embryonic death rate in chicks. Science, 110, 533.

Hays F.A., 1951. Sex ratio in chicks at eight weeks and viability. Poult. Sci., 30, 98-99.

Hays F.A., 1952. Sex ratio in Rhode Island Red chicks at hatching. Poult. Sci., 31, 1094

Hays F.A., Spear E.W., 1950. Embryonic death rate and sex ratio in chicks. Science, 112, 792.
Hays F.A., Spear E.W., 1952. Relation of age of parents to mortality and sex ratio of chicks at eight weeks. Poult. Sci. 31, 792-795.

Heinsohn R., Legge S. Barry S., 1997. Extreme bias in sex allocation in Electus parrots. Proc. R. Soc. B., 264, 1325-1329.

Ichinoe K., 1973. The relationship between hatching time and sex-ratio in chicks. Poult. Sci., 52, 1584-1592.

Kilner R., 1998. Primary and secondary sex ratio manipulation by zebra finches. Anim. Behav., 56, 155-164.

Klein S., Grossman R., Ellendorf F., 2002. Sex diagnosis in early embryos and primary sex ratio development. 11th European Poultry Conference, September 6-10 2002, Bremen Germany Full paper : [CDRom] $\mathrm{E}: \backslash \mathrm{RES} \backslash \mathrm{CGR} \backslash 122 \backslash \mathrm{RES} \backslash 438 . p d f \quad 6 p$. abstract: Arch Geflügelk., 66, $n^{\circ} 6.3 .3,57$.

Komdeur J., Daan S., Tinbergen J, Materman C., 1997. Extreme adaptative modification in sex ratio of the Seychelles warbler's eggs. Nature, 385, 522-525.

Landauer W., Landauer A.B., 1931. Chick mortality and sex ratio in the domestic fowl. Am. Nat., 65, 492-501.

Landauer W., 1957. Primary sex ratio of fowl. Nature, $180,1139-1140$

Mayr E. 1939. The sex ratio in wild birds. Am. Nat., 73, 156-179.

Müller W., Eising C.M., Dikkstra C., Groothuis T.G.G., 2002. Sex differences in yolk hormones depend on maternal social status in Leghorn Chickens (Gallus gallus domesticus). Proc. R. Soc. Lond. B., 269, 2249-2255.

Nager R.G., Monaghan P., Griffiths R., Houston D.C. Dawson R., 1999. Experimental demonstration that offspring sex ratio varies with maternal condition. Proc. Natl. Acad. Sci. USA, 96, 570-573.

Saitoh Y., Saitoh H., Ohtomo K., Mizuno S., 1991 Occupancy of the majority of DNA in the chicken W chromosome by bent repetitive sequences. Chromosoma, 101, 2-40.

Sheldon B.C., 1998. Recent studies of avian sex ratios. Heredity, 80, 397-402.

Schawbl H., 1993. Yolk is a source of maternal testosterone for developing birds. Proc. Natl. Acad. Sci. USA, 90, $11446-11450$

Stefos K., Arrighi F.E., 1971. Heterochromatic nature of W chromosome in birds. Exp. Cell Res., 68, 228-231.

Trivers R.L., Willard D.E. 1973. Natural selection of parental ability to vary the sex ratio of offspring. Science, 179, 90-92.

Van Middelkoop J.H., Lourens S., 2002. Influence of genotype environmental interaction on embryonic mortality and sex ratio Bremen, Abstr 66 (1.5.3):31-32. Full paper [CD Rom] E:/RES/CGR/122/RES/79.pdf, 4p.

West S.A., Reece S.E., Sheldon B.C., 2002. Sex ratios Heredity, 88, 117-124.

Williams G.C., 1979. The question of adaptive sex ratio in outcrossed vertebrates. Proc. R. Soc.Lond. B., 205, 567-580.

Abstract

\section{Sex-ration in wild and domestic birds}

Sex chromosomes determine the sex of embryos in all superior vertebrates including birds. Based on the Mendel laws of segregation of characters, progeny sex ratio in a given population is theoretically equilibrated. However,, examples of unbalanced sex ratio have already been described both in wild and domesticated avian species, suggesting that females in these species may influence the distribution of sex chromosomes, or favour embryo development of one rather than the other sex. Here, we review the circumstances under
\end{abstract}

which female-induced control of genetic sex allocation to the progeny may occur. While a direct demonstration of this phenomenon remains to be established, accessing the underlying mechanisms involved in this type of regulation would provide access to the poultry specialist's old dream of producing females only in layer type chickens and males only in meat-type chickens and mule ducks...

BATELLIER F.,GOVOROUN M., BRILLARD J.P., 2004. Sex-ratio chez les oiseaux sauvages et domestiques. INRA Prod. Anim., 17, 365-372. 